\title{
A Miniature Resonant and Torsional Magnetometer Based on Lorentz Force
}

\author{
Lingqi Wu, Zheng Tian, Dahai Ren * and Zheng You \\ State Key Laboratory of Precision Measurement Technology and Instruments, \\ Department of Precision Instrument, Tsinghua University, Beijing 100084, China; \\ wlq03@mails.tsinghua.edu.cn (L.W.); tz13@tsinghua.org.cn (Z.T.); yz-dpi@tsinghua.edu.cn (Z.Y.) \\ * Correspondence: rendh@tsinghua.edu.cn; Tel.: +86-627-76000
}

Received: 28 November 2018; Accepted: 12 December 2018; Published: 17 December 2018

\begin{abstract}
A microelectromechanical system (MEMS) torsional resonant magnetometer based on Lorentz force was investigated, consisting of torsional structures, torsional beams, metal plates, a coil, and a glass substrate. The Lorentz force, introduced by the interaction between the current in the MEMS coil and an external horizontal magnetic field, leads to displacement of the torsional structure. The strength of the magnetic field is proportional to this displacement, and can be detected with two sensing capacitors fabricated on the torsion structure and the substrate. To improve sensor sensitivity, a folded torsional beam and a double-layer excitation coil were introduced. The fabrication processes included lift-off, anodic bonding, chemical mechanical planarization, silicon nitride (SiNx) deposition, plasma-enhanced chemical vapor deposition, and inductively coupled plasma release. The prototype of the magnetometer was finished and packaged. The sensor performance, including its sensitivity and repeatability, was tested in a low-pressure environment. Additionally, the influences of structural parameters were analyzed, including the resistance of the excitation coil, the initial value of the capacitors, the elastic coefficient of the torsional beam, and the number of layers in the excitation coil. The test results demonstrated that this sensor could meet the requirements for attitude determination systems in low earth orbit satellites.
\end{abstract}

Keywords: magnetometer; MEMS; resonance; Lorentz force; attitude determination

\section{Introduction}

Magnetometers are widely employed in geodesic surveys and aircraft attitude control systems as geomagnetic field detecting sensors [1-3]. As one of the most critical components in the attitude determination systems of spacecraft, they need to be both highly sensitive and accurate. However, the measurement range of the magnetometers can be less than that of sensors used on earth [4-7]. Additionally, miniaturization is important for the application of magnetometers in small satellites [8-11]. Compared to microelectromechanical system (MEMS) magnetometers based on the fluxgate effect [12,13], Hall effect [14,15], and magnetoresistance [16,17], microtorsional resonant magnetometers based on the Lorentz force have the advantages of a high quality factor and high reliability [18-21]. Meanwhile, its MEMS fabrication process is relatively simple [22,23]. Many Lorentz force-based sensors have high requirements for the sensitivity of capacitance measurement, which must be better than $10 \mathrm{aF}$ [24-26]. Combining this with new fabrication methods or other sensors, more new devices were developed [27,28]. In this paper, a MEMS torsional resonant magnetometer based on the Lorentz force was studied, and the relevant parameters were optimized. 


\section{Principles and Simulations}

The MEMS structures of the magnetometer include torsional structures, torsional beams, metal plates, a coil, and a glass substrate (Figure 1). The Lorentz force is introduced by the current in the coil and the external horizontal magnetic field, and leads to differential displacement of the torsional structure, which can be detected with two sensing capacitors fabricated on the torsional structure. The torsion is along the $y$ axis. All the rotation direction conforms to the right-hand rule.

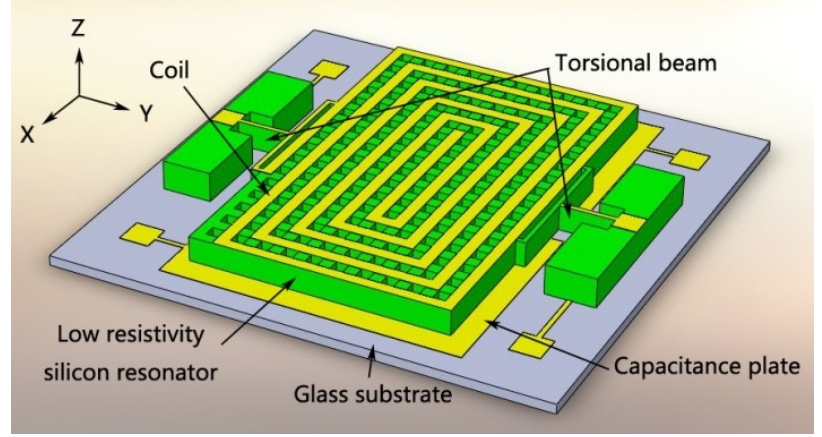

Figure 1. Principle of the microelectromechanical system (MEMS) torsional resonant magnetometer.

Assuming that $k$ is the elastic coefficient of the torsional beam, and $\Theta$ is half the rotational inertia of the torsional structures, then after derivation, the intrinsic frequency and critical damping of the resonance system could be obtained as follows:

$$
\begin{aligned}
& \omega=\sqrt{k / \Theta,} \\
& c=2 \Theta \alpha \omega,
\end{aligned}
$$

where $\alpha$ is the damping ratio of the torsional system; $k$ is a function of the length, width of the torsional beam, and the width and height of the torsional structure; and $\Theta$ is a function of the above parameters and the density of the torsional beam material.

To increase the sensitivity of the sensor, a structure of folded torsional beams and a double-layer excitation coil was designed based on simulation and testing results for the structures of a preliminary fabrication. The structural parameters were also optimized.

Based on the principle and structure of the sensor, maximum system sensitivity can be achieved when the excitation signal frequency is in accordance with the resonance frequency of the torsional system. Using the small signal alternating current (AC) sweep analysis in the Architect module of CoventorWare, the endpoint displacement of the torsional beams was obtained along three perpendicular directions and around three axes (Figure 2). It can be seen that the resonant frequencies in the $z$ and ry directions were very similar (about $1100 \mathrm{~Hz}$ ). However, the resonant frequencies in the $x, y$, and $r z$ directions were approximately $5 \mathrm{kHz}$ to $6 \mathrm{kHz}$, which is far from $1100 \mathrm{~Hz}$. With appropriate excitation signal frequencies, the vibrations in these directions could be suppressed. There was no peak in the vibration in the $r x$ direction. When the frequency was less than $1100 \mathrm{~Hz}$, its attenuation was approximately $-150 \mathrm{~dB}$.

Simulations indicated that the resonant frequencies in the $r y$ and $z$ directions were roughly the same, and there was no resonance peak in the $r x$ direction. This is because its endpoint also vibrated in the $z$ direction (vertical movement) when the torsional pendulum vibrated in the ry direction (around the $y$ axis). The intensity in $r y$ was approximately $-54.3 \mathrm{~dB}$ at $1100 \mathrm{~Hz}$, and the vibrating amplitude in the $r x$ direction was approximately $-150 \mathrm{~dB}$, which was far less than that of the ry direction. Therefore, the torsional vibration around the $y$ axis achieved its maximum intensity when the excitation signal frequency was the same as the resonant frequency of $r y$. However, the vibration amplitudes in the other 
directions were far less than those in this direction. From the perspective of the resonant frequency, these results demonstrated the rationality of the structure design.

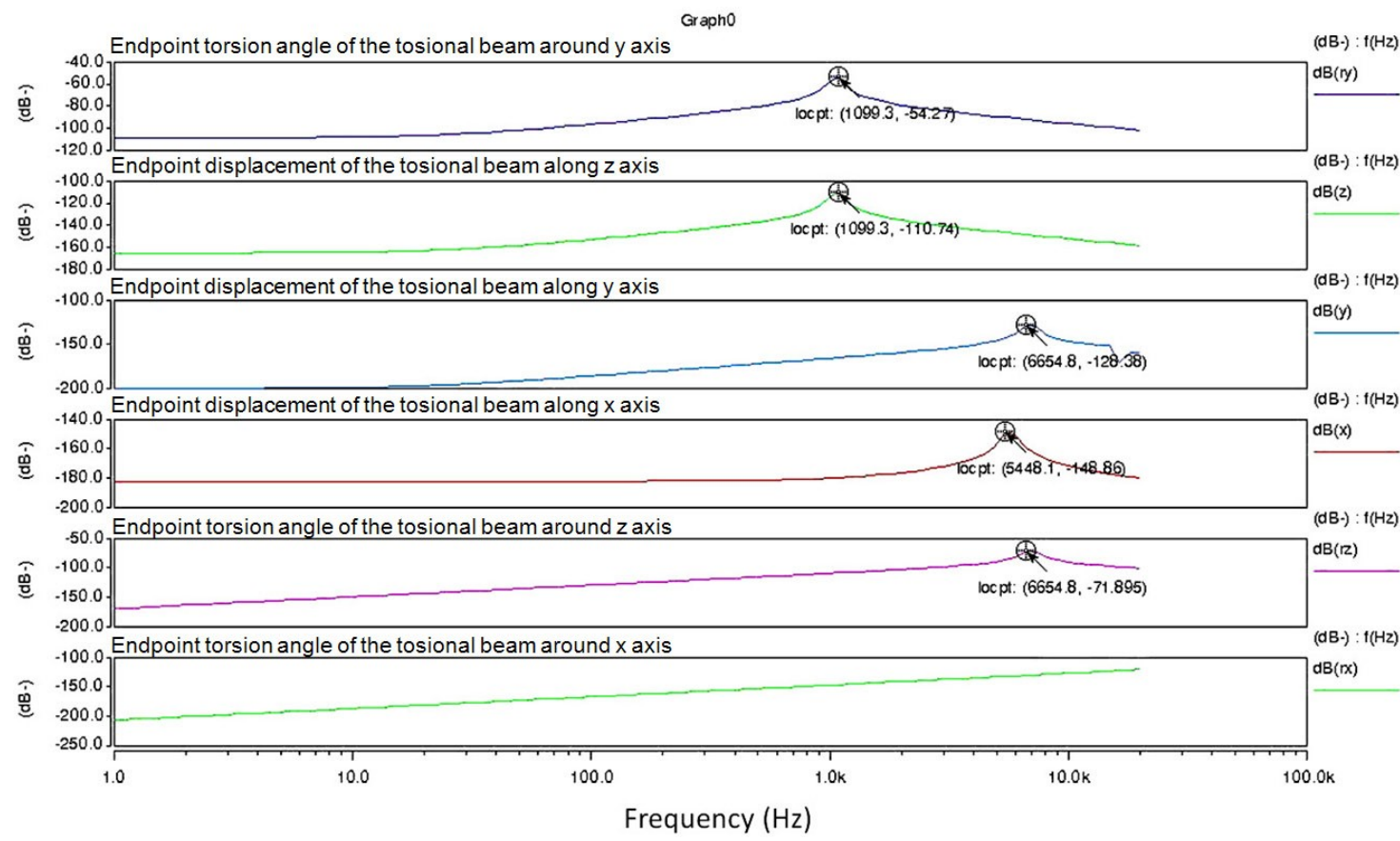

Figure 2. Simulation results for the resonant frequencies of the magnetometer in different directions, indicating the endpoint displacement of the torsional beams along three perpendicular directions and around three axes. Reproduced with permission from [29], published by IEEE, 2013.

\section{Fabrication}

The MEMS fabrication process included dry etching, a lift-off process, anodic bonding, chemical mechanical planarization (CMP), physical vapor deposition, electroplating, and inductively coupled plasma (ICP) etching. The materials used in the microfabrication process were a Pyrex glass substrate and a low-resistivity silicon wafer with a diameter of 4 inches and a thickness of $200 \mu \mathrm{m}$.

The optimized fabrication process is shown in Figure 3. First, a step was etched on the backside of the low-resistivity silicon for glass-silicon bonding, as shown in Figure 3a. Then, the metal plates were fabricated on the glass substrate with the lift-off process, as shown in Figure 3b. Subsequently, a low-resistivity silicon wafer was attached to the glass substrate through anodic bonding (Figure 3c), and the silicon wafer was polished using CMP (Figure 3d). Then, silicon nitride (SiNx) was deposited for insulation (Figure 3e), and gold was patterned to fabricate the coil layer using plasma-enhanced chemical vapor deposition (PECVD) (Figure $3 \mathrm{f}-\mathrm{g}$ ). Finally, the torsional structures were released with ICP, as shown in Figure 3h. 


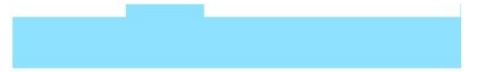

(a)

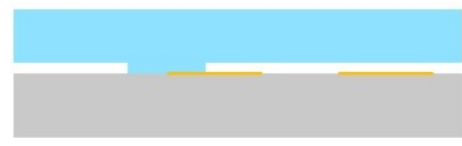

(c)

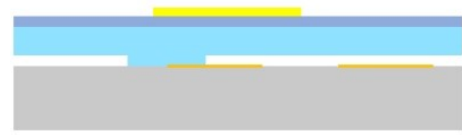

(e)

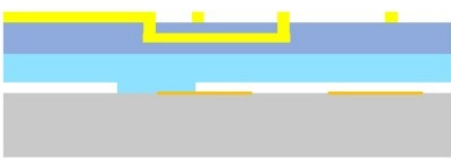

(g)

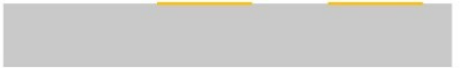

(b)

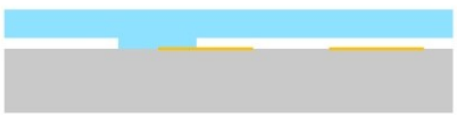

(d)

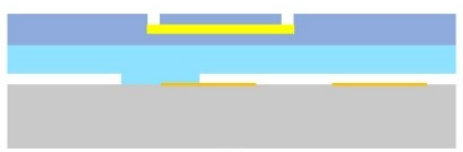

(f)

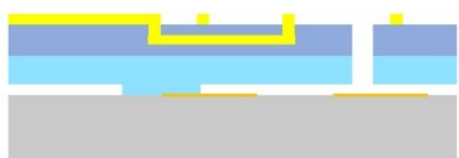

(h)

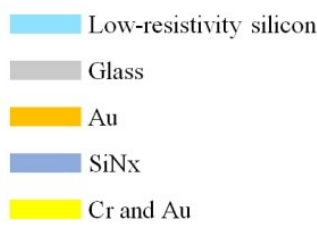

Figure 3. Fabrication process for the MEMS structures: (a) A step was etched on the backside of the low-resistivity silicon for glass-silicon bonding. (b) Fabrication of the metal plates on the glass substrate with the lift-off process. (c) The low-resistivity silicon was attached to the glass substrate through anodic bonding. (d) The silicon wafer was polished using chemical mechanical planarization (CMP). (e) Gold was patterned to fabricate the lead wire. (f) Silicon nitride (SiNx) was deposited for insulation. (g) Gold was patterned to fabricate the coil layer using plasma-enhanced chemical vapor deposition (PECVD). (h) The torsional structures were released with inductively coupled plasma (ICP).

Based on preliminary fabrications and tests, the thickness of the low-resistivity silicon wafer was further decreased to $60 \mu \mathrm{m}$ with CMP before depositing the SiNx insulating layer. This improvement greatly enlarged the torsional angle and increased the convenience of the subsequent ICP process. Thinning the structure layer effectively increased the success rate of the ICP and ensured good mechanical properties of the torsional beam. However, it also created undesirable structural risks. For example, the back of the silicon wafer has a step structure, which forms a cavity with the glass substrate. The pressure on the silicon wafer during the CMP process may break this structure. Therefore, this cavity area should be reduced to prevent the silicon wafer from being crushed.

To reduce the silicon-gold contact resistance introduced by the bonding process, an etching process was added before silicon-glass bonding to remove the oxide layer on the low-resistivity silicon. With this additional process, the resistance of the torsional structure was approximately $1 \mathrm{k} \Omega$, which was very close to the simulation result. By peeling the torsional structure from the device and measuring it with a probe, its resistance was found to be only $500 \Omega$. This indicates that the silicon-gold contact resistance was reduced to approximately $500 \Omega$. The oxide film etching process produced low-resistivity silicon directly bonded to the gold material, avoiding reduction in the conductivity caused by the oxide layer on the silicon. This benefited the subsequent high-precision measurement of the capacitance.

To increase the sensitivity, the torsional beams of the device were designed as folded beams to reduce the elastic coefficient. In addition, two coil layers were achieved in the devices, which greatly increased the sensitivity without increasing the fabrication difficulty.

Figure 4 shows the fabrication results for the key structures. It can be seen that the folded beam structure was intact, and the sidewall of the torsional structures and coil were of good quality. Damping holes were released completely by ICP, reducing the squeeze film air damping effect. These results indicate that by reducing the thickness of the structural layer, both the fabrication quality and the ICP success rate were improved. 


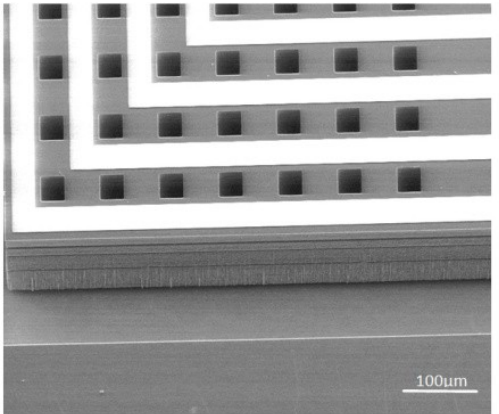

(a)

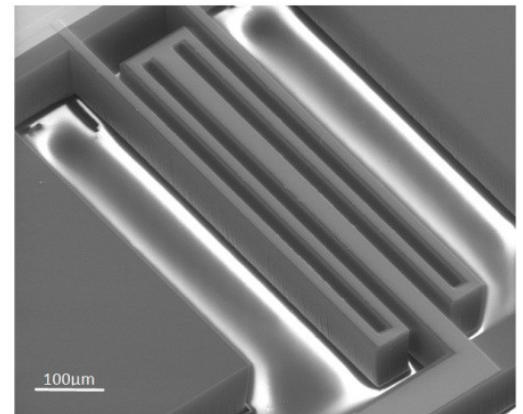

(b)

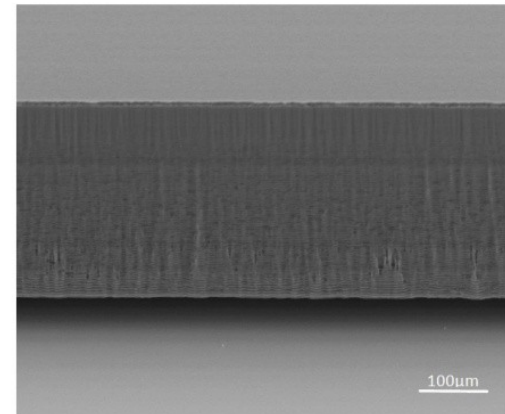

(c)

Figure 4. Fabrication results for the (a) coil, (b) folded beam, and (c) sidewall.

Six gold wires were required for the connections between the gold poles of the shell package and the MEMS structures: Two wires for connections of the excitation coils, two for connections of the capacitor plates, and the remaining two for connections of the torsional pendulums fabricated from the low-resistivity silicon.

There were four gold pillars located at both sides of the tube for bonding. However, there was little Kovar alloy present except for the pins. If the magnetic sensor was well placed for the measurement of a horizontal magnetic field, both the interference and shielding of the external magnetic field caused by the gold pillars could be ignored.

The MEMS structure and the packaged torsional resonant magnetometer are shown in Figure 5a,b.

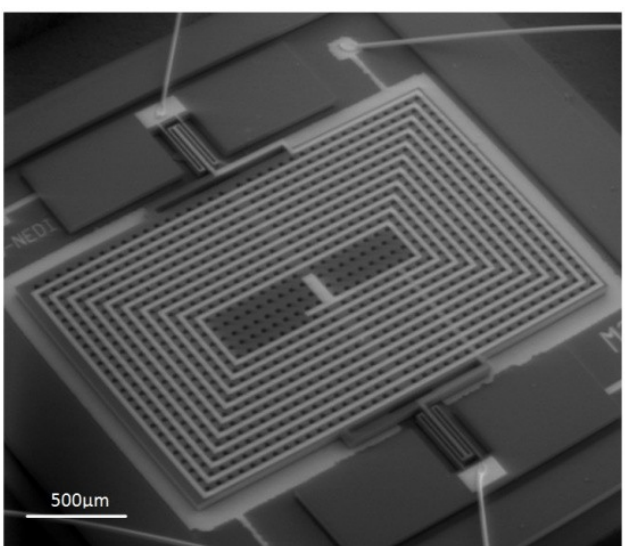

(a)

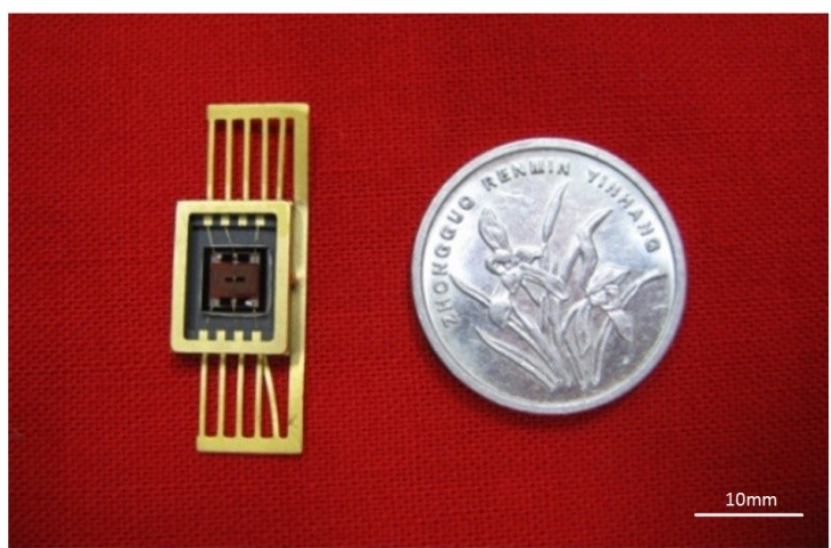

(b)

Figure 5. (a) MEMS structures for the torsional resonant magnetometer; (b) prototype of the MEMS torsional resonant magnetometer.

\section{Driving and Detection Circuit}

The driving and detection circuit of this MEMS torsional resonant magnetometer included two printed circuit boards (PCBs), connected with a pin header. The bottom PCB was a signal carrier generator, which could generate a very stable and accurate carrier signal on the $\mathrm{MHz}$ level. The upper PCB was the miniature magnetometer and a circuit for the signal preprocessing, including a driving circuit, a pre-amplifier circuit, a subsequent amplifier circuit, and a full wave rectifier and demodulator circuit.

The upper PCB had a four-layer structure, where the internal power layer and the Ground (GND) layer were in the middle. Meanwhile, a large number of GND wires were placed on the top and bottom layers to reduce the environmental noises introduced to the pre-amplifier circuit. Because the radiations of the high-frequency carrier signals will cause interferences with other circuits, and the wire of the high-frequency carrier signal is directly connected to the structure of the torsional pendulum, 
this connecting wire should be as short as possible. In this device, the bandwidth was closely related to the quality factor (Q) value. It was about $0.65 \mathrm{~Hz}$ in vacuum and $232 \mathrm{~Hz}$ in air. Figure 6 is the integrated driving circuit and signal conditioning circuit of the MEMS torsional resonant magnetometer.

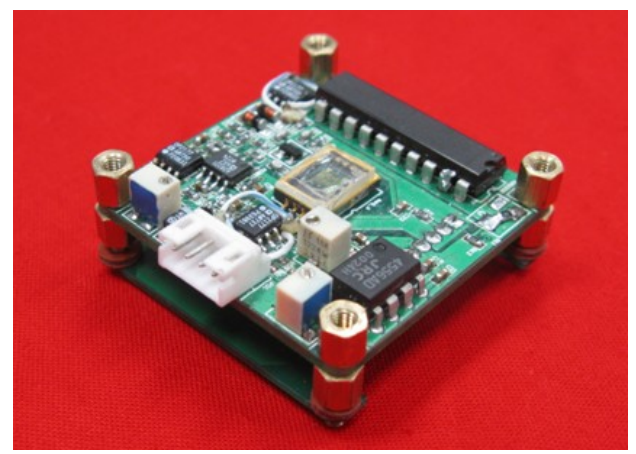

Figure 6. Integrated driving circuit and signal conditioning circuit of the MEMS torsional resonant magnetometer.

\section{Testing and Analysis}

In this magnetometer, the beam length was $500 \mu \mathrm{m}$, and the beam width was $20 \mu \mathrm{m}$. The thickness of the resonator was $60 \mu \mathrm{m}$. The area of the torsional pendulum structure was about $3000 \mu \mathrm{m} \times 2000 \mu \mathrm{m}$. The torsional beam parameters are shown in Table 1. To investigate how relevant parameters influenced device performance, six different types of magnetometers were designed with different MEMS structures. As presented in Table 1, the differences included the shapes and dimensions of the torsional beams, the number of coil turns, the diameter of the damping holes, and the dimensions of the torsional pendulums.

Table 1. Structural parameters of different magnetometer samples.

\begin{tabular}{ccccccc}
\hline $\begin{array}{c}\text { Sample } \\
\text { Number }\end{array}$ & $\begin{array}{c}\text { Torsional } \\
\text { Beam }\end{array}$ & $\begin{array}{c}\text { Coil } \\
\text { Layers }\end{array}$ & $\begin{array}{c}\text { Coil } \\
\text { Turns }\end{array}$ & $\begin{array}{c}\text { Coil Width } \\
(\boldsymbol{\mu m})\end{array}$ & $\begin{array}{c}\text { Damping Hole } \\
\text { Diameter }(\boldsymbol{\mu m})\end{array}$ & $\begin{array}{c}\text { Damping Hole } \\
\text { Quantity }\end{array}$ \\
\hline M1 & Straight-I & Single & 10 & 30 & 30 & 864 \\
M2 & Folded-I & Single & 10 & 30 & 30 & 864 \\
M3 & Folded-II & Single & 6 & 50 & 50 & 364 \\
M4 & Straight-II & Double & 10 & 30 & 30 & 864 \\
M5 & Folded-I & Double & 6 & 50 & 50 & 308 \\
M6 & Folded-II & Double & 6 & 50 & 50 & 308 \\
\hline
\end{tabular}

Here, the difference between the two straight beams was the beam width, $w$, and the differences between the two folded beams were the beam width and the connection length between the main beam and the side beam. There were two types of exciting coil layers, single layer (M1, M2, and M3) and double-layer (M4, M5, and M6). Meanwhile, there were also two different coil arrangements (7 turns with a $50 \mu \mathrm{m}$ width and 11 turns with a $30 \mu \mathrm{m}$ width) for each type.

Except for M3, the size of the torsional pendulums was $3000 \mu \mathrm{m} \times 2000 \mu \mathrm{m}$. For the structural limitation, the samples with the narrow excitation coils had 864 damping holes with a diameter of $30 \mu \mathrm{m}$, and the samples with the wider excitation coils had 364 damping holes with a diameter of $50 \mu \mathrm{m}$.

\subsection{Sensitivity}

The output properties of the different magnetometer models were tested in a magnetic field intensity range of $0-50 \mu \mathrm{T}$ with an energizing current amplitude of $1 \mathrm{~mA}$ and a vacuum of $10 \mathrm{~Pa}$ at room temperature. The test results are shown in Figure 7. For simplicity, the measured voltage was the 
peak-to-peak AC voltage signal without synchronous demodulation, which had the same frequency as the driving signal.

For the different magnetometer types, it was clear that there was a very good linear correlation between the output voltage and the magnetic field intensity. In contrast, their sensitivity differences were obvious. As shown in Table 2, the sensitivity of the M4 model was about 8 times that of the M6 model. This was due to the different MEMS structures and parameters, including the torsional beam structures, torsional beam parameters, coil types, torsional pendulum sizes, and the parameters and quantities of the damping holes.

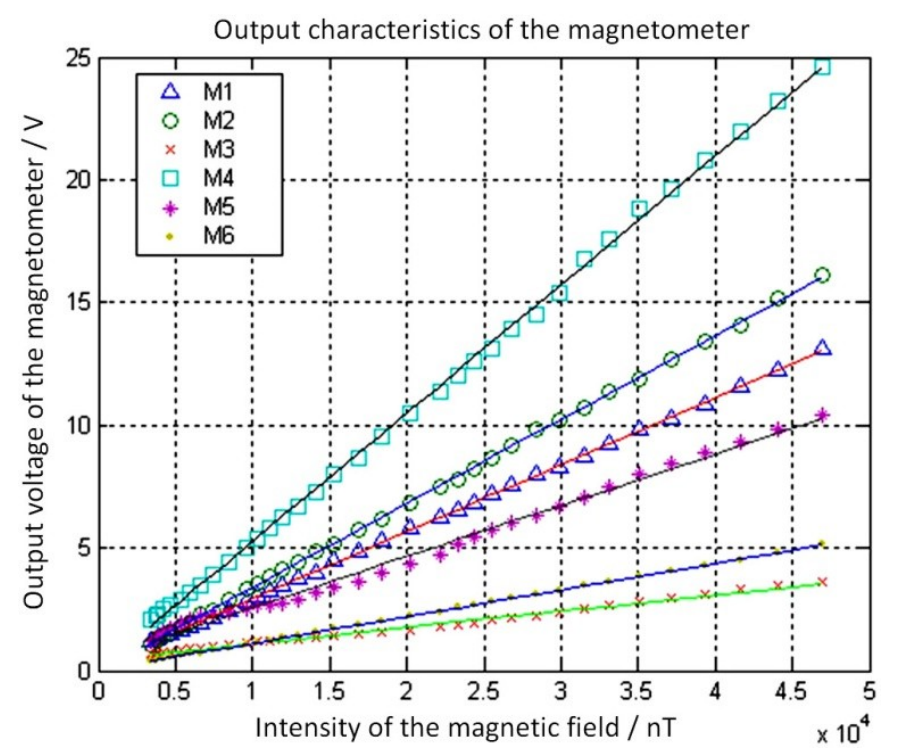

Figure 7. Output properties for different magnetometer models. Reproduced with permission from [29], published by IEEE, 2013.

Table 2. Sensitivity of different device types.

\begin{tabular}{ccc}
\hline Sample Number & $\begin{array}{c}\text { Analytic Expression of the Fitting Curve } \\
\boldsymbol{x}(\mathbf{n T}), \boldsymbol{y}(\mathbf{V})\end{array}$ & Sensitivity \\
\hline M1 & $y=0.000272 x+0.228$ & $272 \mathrm{mV} / \mu \mathrm{T}$ \\
M2 & $y=0.000342 x-0.0287$ & $342 \mathrm{mV} / \mu \mathrm{T}$ \\
M3 & $y=0.000066 x+0.438$ & $66 \mathrm{mV} / \mu \mathrm{T}$ \\
M4 & $y=0.000523 x+0.0489$ & $523 \mathrm{mV} / \mu \mathrm{T}$ \\
M5 & $y=0.000208 x+0.499$ & $208 \mathrm{mV} / \mu \mathrm{T}$ \\
M6 & $y=0.000108 x+0.360$ & $108 \mathrm{mV} / \mu \mathrm{T}$ \\
\hline
\end{tabular}

\subsection{Resonant Frequency and Quality Factor}

With a constant outside magnetic field strength and varying excitation current frequency, amplitude changes were observed in the magnetometer output voltages. The frequency characteristics of the exciting current for the different samples are shown in Figure 8.

Because the vacuum level in the test environment was $10 \mathrm{~Pa}$, the damping ratio, $\alpha$, of the magnetometer torsional pendulum resonant system was close to 0 . Therefore, the quality factor, $Q$, could be obtained as follows:

$$
Q=f_{0} /\left(f_{u}-f_{d}\right),
$$

where $f_{0}$ is the resonant frequency of the torsional pendulum, and $f_{u}$ and $f_{d}$ are the upper and lower frequency limits, respectively, when the output amplitude decreases to $\sqrt{2} / 2(-3 \mathrm{~dB})$ of the peak. 
From Equation (3) and Figure 9, the resonant frequency and the quality factor for each sample were obtained. As shown in Table 3, the resonant frequency test results were generally in agreement with the theoretical analysis, and the error was about $15 \%$.

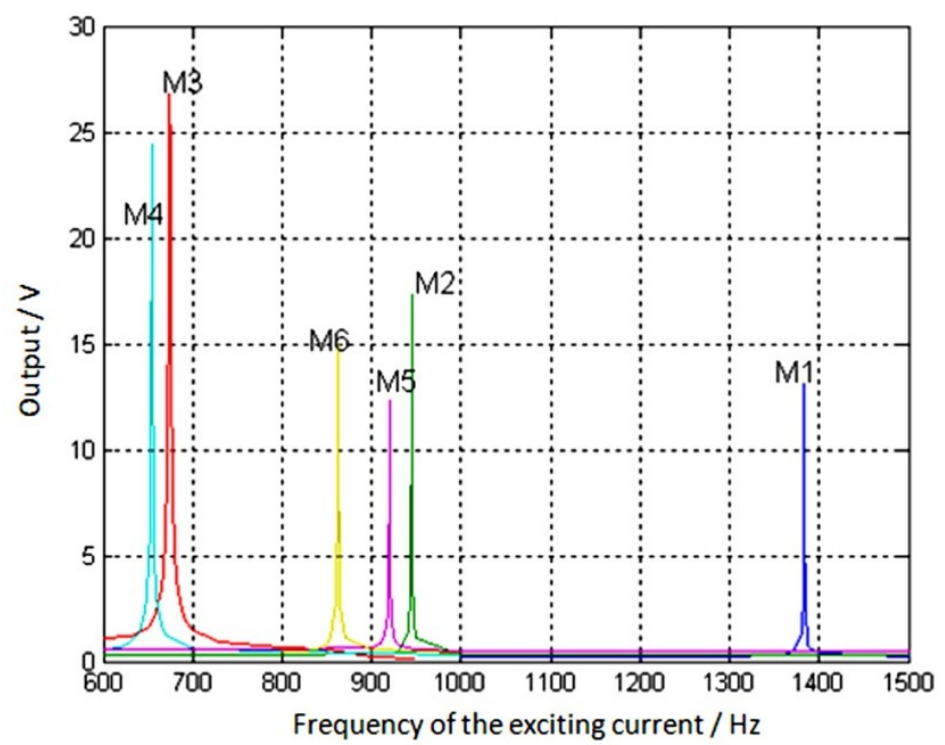

Figure 8. Frequency characteristics of the exciting current for different samples. Reproduced with permission from [29], published by IEEE, 2013.

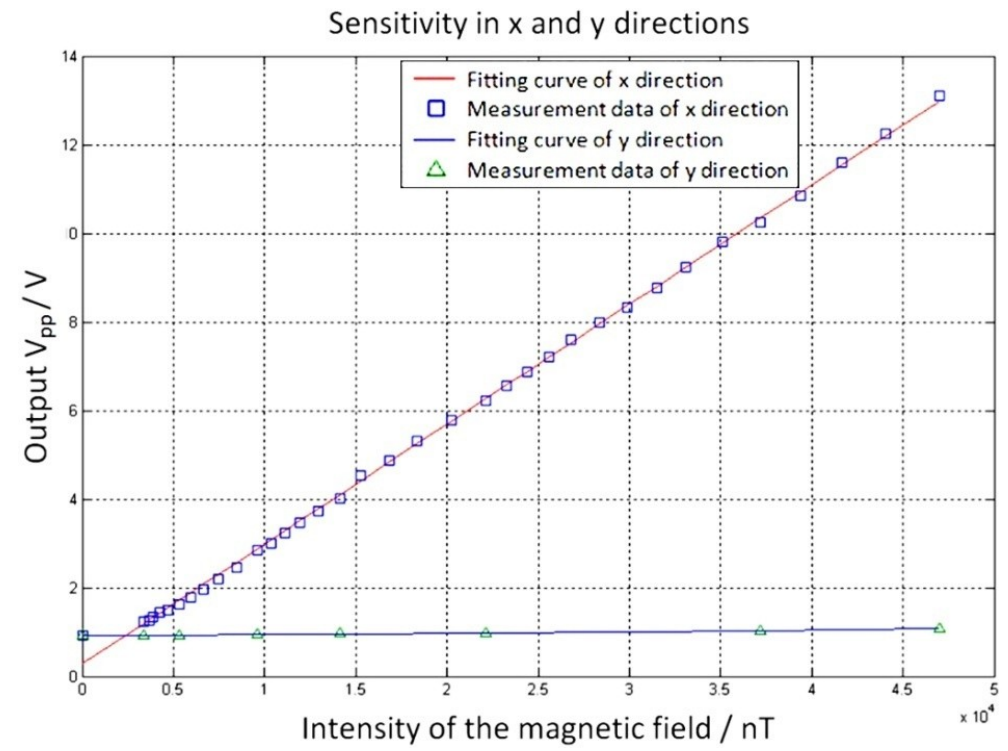

Figure 9. Comparison of the sensitivity in orthogonal axes.

Table 3. Measured and calculated resonance frequency of different magnetometer samples.

\begin{tabular}{cccc}
\hline Sample Number & $\boldsymbol{Q}$ Value & Calculated Frequency $\mathbf{( H z )}$ & Measured Frequency $\mathbf{( H z )}$ \\
\hline M1 & 2530.1 & 1696 & 1383.2 \\
M2 & 1684.5 & 1047 & 944.8 \\
M3 & 317.0 & 798 & 674.3 \\
M4 & 485.9 & 868 & 654.4 \\
M5 & 1132.6 & 1021 & 919.9 \\
M6 & 1038.5 & 970 & 862.4 \\
\hline
\end{tabular}




\subsection{Analysis of the Structural Parameters}

The parameters of different magnetometer types were analyzed, and their performance was compared to verify the parameter choices.

\subsubsection{Resistance of the Excitation Coil}

Ten samples of each type were selected, and the coil resistance was measured. The results showed that the resistance of the double coils was twice that of a single coil with the same number of turns and width. As the upper and lower coils were approximately the same length, it was reasonable that the resistance of the double coil layer was approximately twice that of the single coil layer. This indicates that the fabrication based on the novel design was successful. There were no short circuiting phenomena between the upper and the lower coils due to low fabrication quality of the SiNx insulating layer. According to the measurement results, the double coil process resulted in a high yield. In Table S3, we list the measured and calculated coil resistances.

\subsubsection{Initial Value of the Capacitor Polar Plate}

The initial capacitor value, $\mathrm{C} 0$, between the torsional structure and the capacitor polar plate beneath was simulated by CoventorWare, resulting in a value of $2.663 \mathrm{pF}$. It was also measured with a capacitance-voltage $(\mathrm{C}-\mathrm{V})$ characteristic tester, with results ranging from $2.6 \mathrm{pF}$ to $2.7 \mathrm{pF}$, which was in good agreement with the simulation result.

\subsubsection{Elastic Coefficient of the Torsional Beam}

When other structural parameters remained the same, the sensitivity of the M1-type device with a straight beam (width of $25 \mu \mathrm{m}$ ) was $272 \mathrm{mV} / \mu \mathrm{T}$, whereas the M2-type device with the folded beam (main beam width of $30 \mu \mathrm{m}$, side beam width of $30 \mu \mathrm{m}$ ) was $342 \mathrm{mV} / \mu \mathrm{T}$. The sensitivity ratio was thus $K_{2} / K_{1} \approx 1.26$.

The simulation analysis showed that the capacitance variance of M1 was $\Delta C_{1}=2.94 \mathrm{fF}$, while that of M2 was $\Delta C_{2}=4.15 \mathrm{fF}$ with an exciting current of $30 \mathrm{~mA}$ and a magnetic field intensity of $50 \mu \mathrm{T}$. For this type of magnetometer, the ratio of the capacitance variance amplitudes, the ratio of the magnetometer output voltage amplitudes, and the ratio of the sensitivities were the same. Based on the simulation results, the sensitivity ratio of these two devices was $\Delta C_{2} / \Delta C_{1} \approx 1.41$, which agreed well with the experimental data. In addition, it can be seen that the sensitivity of the folded beam device was 1.3 times that of the straight beam device.

Because the excitation coil was led out along the torsional beam, the excitation coil and the torsional beam had the same width. However, the equivalent width of the folded beam was different from that of the straight beam, making their allowable currents different. According to previous experience with the MEMS process, the maximum allowable current of a gold coil with a sectional size of $1 \mu \mathrm{m} \times 1 \mu \mathrm{m}$ was $4 \mathrm{~mA}$, which was proportional to the coil width and thickness. Due to the difference in the equivalent width, the maximum allowable current ratio of the folded beam type and the straight beam type device was approximately $1.2(30 / 25)$. The current amplitude was approximately proportional to the amplitude of the capacitance variance under small angle torsion. Additionally, the sensitivity of the folded beam type was approximately $1.6(1.3 \times 1.2)$ times that of the straight beam type at the maximum allowable current. Thus, the sensitivity was clearly improved with the folded beam structure.

For comparison of straight beam-type devices with different widths (M1 and M4), from Equation (1), $\omega=\sqrt{k / \Theta}$ was obtained. The moment of inertia $(\Theta)$ was the same for the two devices, and the elastic coefficient of a straight beam, $k$, was proportional to $w^{3} \gamma$, where $w$ and $\gamma$ are the width of the straight beam and a coefficient related to $w / h$, respectively. For the M1 device, $w / h \approx 0.417$, and $\gamma$ 
was 0.254 . For the M4 device, $w / h \approx 0.25$, and $\gamma$ was 0.282 [30]. Therefore, the theoretical resonance frequency ratio between M1 and M4 could be obtained as follows:

$$
\frac{f_{1}}{f_{4}}=\frac{\omega_{1}}{\omega_{4}}=\sqrt{\frac{k_{1}}{k_{4}}}=\sqrt{\frac{w_{1}^{3} \gamma_{1}}{w_{4}^{3} \gamma_{4}}}=\sqrt{\frac{25^{3} \times 0.254}{15^{3} \times 0.282}} \approx 2.04
$$

The practical frequency ratio of the two devices was $f_{c 1} / f_{c 4}=1383.2 / 654.4 \approx 2.11$, which agreed well with the theoretical results. According to Equation (3), the elastic coefficient of the torsional beam was proportional to the cube of the beam width. Therefore, decreasing the width could notably decrease the elastic coefficient of the torsional beam, thus increasing the torsional angle under the same torque.

\subsubsection{Layer Quantity of the Exciting Coil}

According to the analysis, the torque generated by the magnetic sensor was the sum of the torques generated by each turn of the exciting coil perpendicular to the magnetic field. In the double coil structure, the number of exciting coils in that direction was twice that of the single coil structure. Therefore, the torque amplitude, $M$, was theoretically twice that of the single coil structure. The maximal twisting angle of the magnetometer torsional pendulum could be calculated as follows:

$$
\varphi_{r}=\frac{M}{k} Q
$$

where $Q$ is the quality factor. Analysis of the performance of the capacitance measurement circuit showed that the amplitude of the output voltage was directly proportional to the capacitance variance amplitude, $\Delta C$, and thus was also directly proportional to the maximal twisting angle, $\varphi_{r}$, of the torsional pendulum when the twisting angle was small. The M1-type device had a single exciting coil with a torsion beam width of $w_{1}=25 \mu \mathrm{m}, Q=2350.1$, and sensitivity $=272 \mathrm{mV} / \mu \mathrm{T}$. The M4-type device had a double exciting coil with a torsion beam width $w_{4}=15 \mu \mathrm{m}, Q=485.9$, and sensitivity $=$ $523 \mathrm{mV} / \mu \mathrm{T}$. Other than these previously mentioned differences, these two device types had the same structures. The structural parameters and test data for the M1- and M4-type devices were input into Equation (4) to obtain the ratio between the torques of the M4 exciting coil and the M1 exciting coil as follows:

$$
\frac{M_{4}}{M_{1}}=\frac{\varphi_{r 4} Q_{1} k_{4}}{\varphi_{r 1} Q_{4} k_{1}}=\frac{K_{4} Q_{1} k_{4}}{K_{1} Q_{4} k_{1}}=\frac{523 \times 2350.1 \times 15^{3} \times 0.282}{272 \times 485.9 \times 25^{3} \times 0.254} \approx 2.23
$$

According to the theoretical analysis, the expected torque ratio between the M4-type and M1-type devices was 2, which was basically consistent with the test value of 2.23. This indicates that the design of the double exciting coil structure was effective, and the increased excitation torque of the device was approximately double the original value. Analysis of the device linearity showed that its sensitivity was also double the original value.

\subsection{Sensitivity in the Orthogonal Axis}

This magnetometer could only sense the magnetic field in the horizontal plane perpendicular to the torsion beam (the $x$ axis in Figure 1). To investigate its sensitivity in the orthogonal axis, the sensitivity was tested in the $x$ and $y$ directions. From Figure 9, the sensitivity along the $x$ axis and $y$ axis was $K_{x}=270 \mathrm{mV} / \mu \mathrm{T}$ and $K_{y}=3.25 \mathrm{mV} / \mu \mathrm{T}$. The sensitivity in the $x$ direction was about 83 times that in the $y$ direction. Therefore, the sensor could be regarded as a monoaxial sensitive sensor.

\subsection{Repeatability}

The M3-type device was selected to test the repeatability of the output characteristics. With a pressure intensity of $P=5 \mathrm{~Pa}$, an excitation voltage frequency of $f_{0}=673.9 \mathrm{~Hz}$, and an excitation voltage peak value of $V_{\mathrm{pp}}=300 \mathrm{mV}$, the intensity of the external magnetic field was adjusted three 
times from $0 \mathrm{nT}$ to 30,000 nT to investigate the change in output voltage with each adjustment of the magnetic field intensity. Linear fitting was performed for each of the three measurements, and the resulting curves are shown in Figure 10.

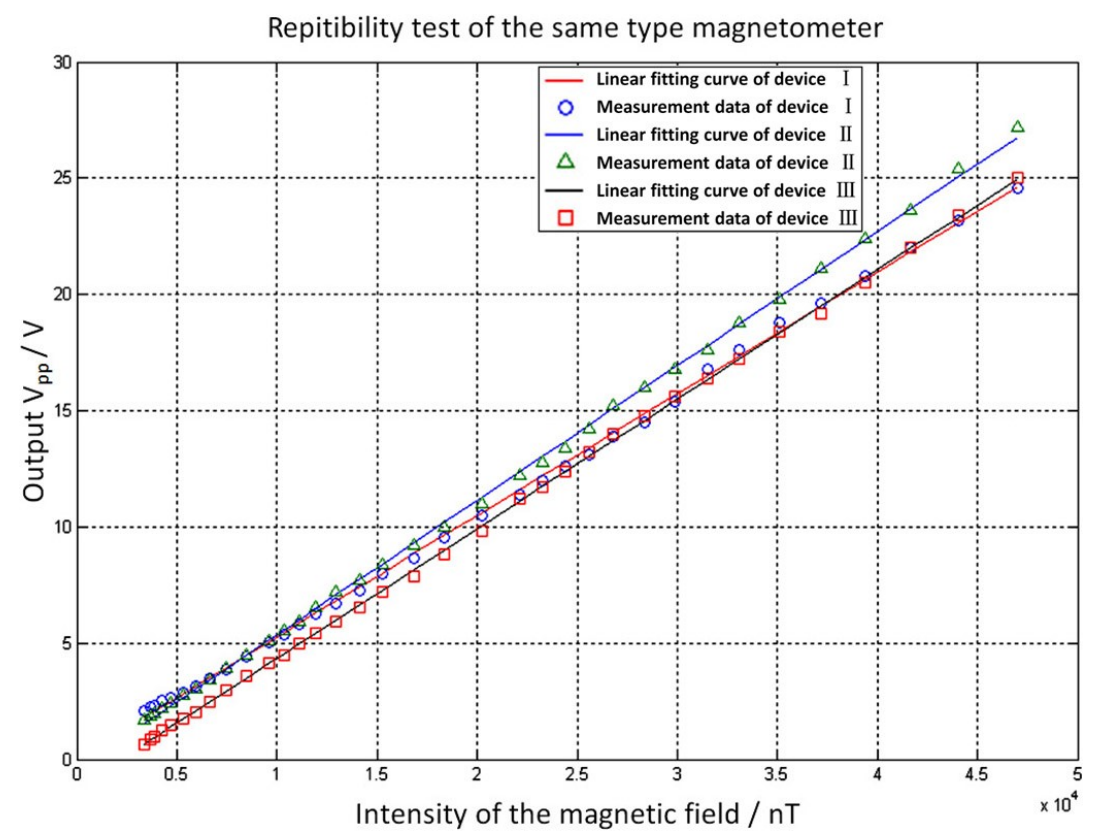

Figure 10. Linear fitting curves for measurement data of the micro magnetometer.

The performances reflected by the linear fitting curves for the three measurements are summarized in Table 4. The linear correlation coefficient of the data and the sensitivity of the fitting curve were very close, indicating good repeatability.

Table 4. Results of the three measurements.

\begin{tabular}{cccc}
\hline Serial Number & Linearly Dependent Coefficient $\boldsymbol{r}$ & $\begin{array}{c}\text { Sensitivity } \boldsymbol{K} \\
(\mathbf{m V} / \boldsymbol{\mu} \mathbf{T})\end{array}$ & $\begin{array}{c}\text { Analytical Expression } \\
\boldsymbol{x}(\mathbf{n T}), \boldsymbol{y} \mathbf{( V )}\end{array}$ \\
\hline 1 & 0.9970 & 461 & $y=0.000461 x+0.0294$ \\
2 & 0.9939 & 454 & $y=0.000454 x+0.0359$ \\
3 & 0.9962 & 469 & $y=0.000469 x+0.00319$ \\
\hline
\end{tabular}

Next, the main causes of repeatability error were analyzed when the magnetometer was placed in a magnetic field with a variable external magnetic field intensity. As some of the components on the circuit board and the package shells contained nickel, iron, cobalt, and the Kovar alloy compounded from such materials, these components were prone to magnetization and would generate a static magnetic field of a given intensity. When the strength of the magnetic field changed, these materials were also magnetized with an altered strength, and the influence of the magnetic field they generated would also vary. This caused the sensor to output different values for a magnetic field with the same strength during different measurements, thus decreasing the repeatability of the sensor.

Meanwhile, during multiple measuring processes with the same magnetometer, the zero deviations of the prototype were recorded for each measurement. The resulting curve is shown in Figure 11. The zero deviations of the prototype ranged between $0.40 \mathrm{~V}$ and $0.44 \mathrm{~V}$, with an average of $0.413 \mathrm{~V}$. The standard deviation was approximately $14 \mathrm{mV}$. This standard deviation was very small relative to the output amplitudes, which satisfied the repeatability requirement. 


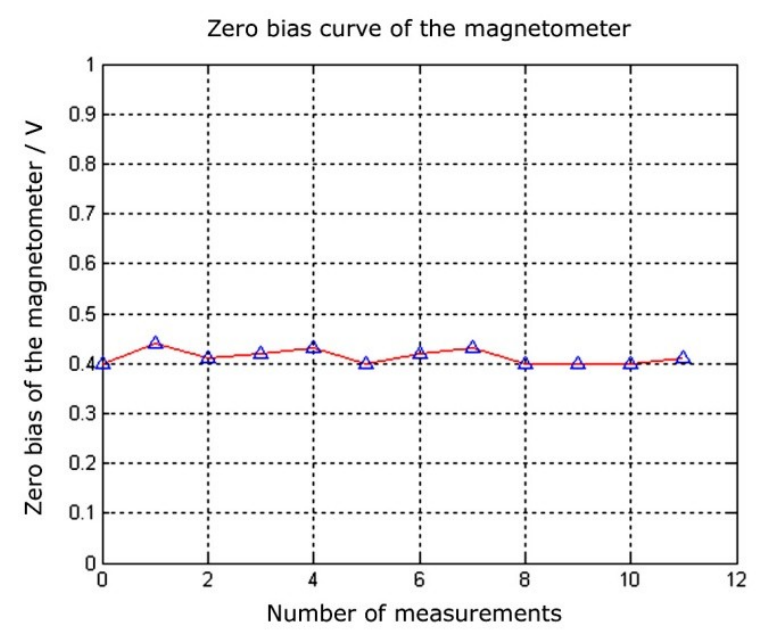

Figure 11. Testing curve for the zero deviations of the prototype.

\subsection{Noise}

Noise was one of the most important parameters of the sensor. Figure 12 shows the noise spectrum of the sensor. The inset shows a typical time trace. Its noise peak-to-peak value was $60 \mathrm{mV}$. Since the sensitivity of the sample in this range was $1 \mathrm{~V} / \mu \mathrm{T}$, the noise was $60 \mathrm{nT} / \sqrt{\mathrm{Hz}}$.

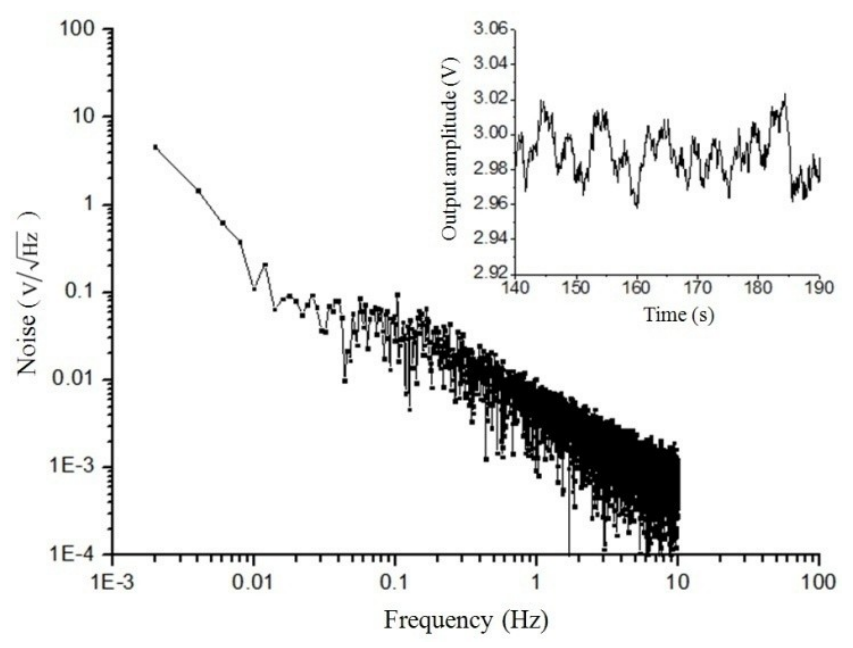

Figure 12. Test result of the noise power spectral density.

\section{Conclusions}

A MEMS torsional resonant magnetometer was designed based on the Lorentz force. MEMS processes were analyzed and optimized based on fabrication experiments and tests. Detailed tests were conducted on the prototypes to verify the choice of design parameters. Experimental measurements showed that the capacitance change could reach $100 \mathrm{fF}$ under the condition of a $10 \mathrm{~Pa}$ air pressure when an external magnetic field of $50 \mu \mathrm{T}$ was applied. Such sensitivity effectively decreased the difficulties in the signal processing. Experiments indicated that the resolution of the device was better than $30 \mathrm{nT}$. Additional tests demonstrated good repeatability of the measurement process as well as the zero deviations of the device.

To increase the sensitivity, the drive current $I$, coil length $L$, pendulum width $W$, and beam length $l$ should be maximized. At the same time, the plate distance $d_{0}$, beam thickness $h$, beam width $w$, and shear modulus $G$ should be minimized. The quality factor should be as high as possible, which can be realized by increasing the size and the number of holes. 
Compared to other MEMS magnetometers, the structures of this torsional resonant magnetometer were easier to fabricate. The test results demonstrated that it could meet the requirements of attitude determination systems of low earth orbit satellites.

Supplementary Materials: The following are available online at http:/ /www.mdpi.com/2072-666X/9/12/666/s1, Figure S1: The layout of the double coil. (a) The upper coil of the double coil system, and (b) the sublayer of the double coil, connecting the upper layer coil in the center, Figure S2: Circuit principle of the magnetometer, Figure S3: Sketch map of the torsional structure, Table S1: Torsional beam parameters, Table S2: Comparisons of different prototypes, Table S3: Measured and calculated coil resistances of different magnetometer samples.

Author Contributions: L.W. made the fabrications and performed the experiments; Z.T. designed the circuit and performed the experiments; D.R. designed the structures and analyzed the data; Z.Y. designed the test platform.

Acknowledgments: This work is supported by the National High-Tech Research and Development Program of China. We thank the China Electronics Technology Group Corporation, especially Jian Zhu, Yongjun Yang, and Yuanwei Yu for their help on MEMS fabrications.

Conflicts of Interest: The authors declare no conflict of interest.

\section{References}

1. Langfelder, G.; Tocchio, A. Operation of Lorentz-Force MEMS Magnetometers With a Frequency Offset Between Driving Current and Mechanical Resonance. IEEE Trans. Magn. 2014, 50, 4700106. [CrossRef]

2. Zhang, Z.Q.; Yang, G.Z. Micromagnetometer calibration for accurate orientation estimation. IEEE Trans. Biomed. Eng. 2015, 62, 553-560. [CrossRef]

3. Li, X.; Hu, J.; Chen, W.; Yin, L.; Liu, X. A Novel High-Precision Digital Tunneling Magnetic Resistance-Type Sensor for the Nanosatellites' Space Application. Micromachines 2018, 9, 121. [CrossRef]

4. Wu, Y.P.; Ren, D.H.; You, Z. HXMT satellite for space hard X-ray observation. Adv. Space Res. 2004, 34, $2667-2672$. [CrossRef]

5. Abdelrahman, M.; Park, S.-Y. Sigma-Point Kalman Filtering for Spacecraft Attitude and Rate Estimation using Magnetometer Measurements. IEEE Aerosp. Electron. Syst. 2011, 47, 1401-1415. [CrossRef]

6. Brown, P.; Beek, T.; Carr, C.; O’Brien, H.; Cupido, E.; Oddy, T.; Horbury, T.S. Magnetoresistive magnetometer for space science applications. Meas. Sci. Technol. 2012, 23, 1-11. [CrossRef]

7. Tian, Z.; Ren, D.; You, Z. Self-Oscillation-Based Frequency Tracking for the Drive and Detection of Resonance Magnetometers. Sensors 2016, 16, 744. [CrossRef]

8. Díaz-Michelena, M. Small Magnetic Sensors for Space Applications. Sensors 2009, 9, 2271-2288. [CrossRef]

9. Coillot, C.; Moutoussamy, J.; Lebourgeois, R.; Ruocco, S.; Chanteur, G. Principle and Performance of a Dual-Band Search Coil Magnetometer: A New Instrument to Investigate Fluctuating Magnetic Fields in Space. IEEE Sensors J. 2016, 10, 255-260. [CrossRef]

10. Topal, U.; Sözeri, H. A Novel Design of Fluxgate Magnetometer: An Insect like Configuration. J. Supercond. Novel Magn. 2015, 28, 711-714. [CrossRef]

11. Laghi, G.C.; Marra, R.; Minotti, P.; Tocchio, A.; Langfelder, G. A 3-D Micromechanical Multi-Loop Magnetometer Driven Off-Resonance by an On-Chip Resonator. J. Microelectromech. Syst. 2016, 25, 637-651. [CrossRef]

12. Snoeij, M.F.; Schaffer, V.; Udayashankar, S.; Ivanov, M.V. Integrated Fluxgate Magnetometer for Use in Isolated Current Sensing. IEEE J. Solid-State Circuits 2016, 51, 1684-1694. [CrossRef]

13. Trigona, C.; Sinatra, V.; Andò, B.; Baglio, S.; Bulsara, A.R. Flexible Microwire Residence Times Difference Fluxgate Magnetometer. IEEE Trans. Instrum. Meas. 2017, 66, 559-568. [CrossRef]

14. Grosz, A.; Mor, V.; Amrusi, S.; Faivinov, I.; Paperno, E.; Klein, L. A High-Resolution Planar Hall Effect Magnetometer for Ultra-Low Frequencies. IEEE Sensors J. 2016, 16, 3224-3230. [CrossRef]

15. Crescentini, M.; Marchesi, M.; Romani, A.; Tartagni, M.; Traverso, P.A. A Broadband, On-Chip Sensor Based on Hall Effect for Current Measurements in Smart Power Circuits. IEEE Trans. Instrum. Meas. 2018, 67, 1470-1485. [CrossRef]

16. Denoual, M.; Saez, S.; Kauffman, F.; Dolabdjian, C. Magnetorelaxometry using improved giant magnetoresistance magnetometer. Sens. Actuators, A 2010, 159, 184-188. [CrossRef]

17. Luong, V.S.; Jeng, J.T.; Lu, C.C.; Hsu, H.Y. Low-noise tunneling-magnetoresistance vector magnetometers with flux chopping technique. Measurement 2017, 109, 297-303. [CrossRef] 
18. Li, M.; Sonmezogl, S.; Horsley, D.A. Extended Bandwidth Lorentz Force Magnetometer Based on Quadrature Frequency Modulation. J. Microelectromech. Syst. 2015, 24, 333-342. [CrossRef]

19. Ghosh, S.; Lee, E.Y. A Lorentz force magnetometer based on a piezoelectric-on-silicon square-extensional mode micromechanical resonator. Appl. Phys. Lett. 2017, 110, 253507.

20. Herrera-May, A.L.; García-Ramírez, P.J.; Aguilera-Cortés, L.A.; Figueras, E.; Martínez-Castillo, J.; Manjarrez, E.; Sauceda, A.; García-González, L.; Juárez-Aguirre, R. Mechanical design and characterization of a resonant magnetic field microsensor with linear response and high resolution. Sens. Actuators A 2011, 165, 399-409. [CrossRef]

21. Li, M.; Rouf, V.T.; Thompson, M.J.; Horsley, D.A. Three-Axis Lorentz-Force Magnetic Sensor for Electronic Compass Applications. J. Microelectromech. Syst. 2012, 21, 1002-1010. [CrossRef]

22. Dominguez-Nicolas, S.M.; Juarez-Aguirre, R.; Herrera-May, A.L.; García-Ramírez, P.; Figueras, E.; Gutierrez-D., E.A.; Tapia, J.A.; Trejo, A.; Manjarrez, E. Respiratory magnetogram detected with a MEMS device. Int. J. Med. Sci. 2013, 10, 1445-1450. [CrossRef]

23. Choi, S.; Yoon, Y.-K.; Kim, S.-H.; Allen, M.G. Nonlinear sensitivity enhancement of resonant microsensors and its application to low power magnetic sensing. J. Micromech. Microeng. 2011, 21, 045004. [CrossRef]

24. Wu, G.; Xu, D.; Xiong, B.; Feng, D.; Wang, Y. Resonant magnetic field sensor with capacitive driving and electromagnetic induction sensing. IEEE Electron Device Lett. 2013, 34, 459-461. [CrossRef]

25. Langfelder, G.; Buffa, C.; Frangi, A.; Tocchio, A.; Lasalandra, E.; Longoni, A. Z-axis magnetometers for MEMS inertial measurement units an industrial process. IEEE Trans. Ind. Electron. 2013, 60, 3983-3990. [CrossRef]

26. Yan, M.Z.; Dong, Z.; Ren, D.H.; You, Z. Research of tortional tunneling magnetometer designing method. Chin. J. Sci. Instrum. 2006, 27, 1154-1158.

27. Elsayed, M.Y.; Cicek, P.; Nabki, F.; El-Gamal, M.N. Surface micromachined combined magnetometer/ accelerometer for above-IC Integration. J. Microelectromech. Syst. 2015, 24, 1029-1037. [CrossRef]

28. Wattanasarn, S.; Matsumoto, K.; Shimoyama, I. 3D Lorentz force magnetic sensor using ultra-thin piezoresistive cantilevers. In Proceedings of the 2013 IEEE 26th International Conference on Micro Electro Mechanical Systems (MEMS), Taipei, Taiwan, 20-24 January 2013.

29. Ren, D.H.; Cui, M.Y.; Wu, L.Q.; Guo, T.W.; Xu, C.T.; You, Z. A MEMS torsional resonant magnetometer for the attitude determination in space. In Proceedings of the 2013 Transducers \& Eurosensors XXVII: The 17th International Conference on Solid-State Sensors, Actuators and Microsystems (TRANSDUCERS \& EUROSENSORS XXVII), Barcelona, Spain, 16-20 June 2013.

30. Selvakumar, A.; Najafi, K. A High-Sensitivity Z-Axis Capacitive Silicon microaccelerometer with a torsional suspension. J. Microelectromech. Syst. 1998, 7, 192-200. [CrossRef] 